\title{
The Role of Magnetic Fields in Spiral Galaxies
}

\author{
Rainer Beck \\ MPI für Radioastronomie, Auf dem Hügel 69, 53121 Bonn, Germany
}

\begin{abstract}
Interstellar magnetic fields are strong: up to $25 \mu \mathrm{G}$ in spiral arms and $40 \mu \mathrm{G}$ in nuclear regions. In the spiral galaxy NGC 6946 the average magnetic energy density exceeds that of the thermal gas. Magnetic fields control the evolution of dense clouds and possibly the global star formation efficiency in galaxies. Gas flows and shocks in spiral arms and bars are modified by magnetic fields. Magnetic forces in star-forming circumnuclear regions are able to drive mass inflow towards the active nucleus. Magnetic fields are essential for the propagation of cosmic rays and the formation of galactic winds and halos.
\end{abstract}

Keywords: Galaxies: spiral - Galaxies: magnetic fields - ISM: magnetic fields

\section{Interstellar Magnetic Fields Are Hard To Observe}

... but it's easier than most people think. In radio continuum the typical degrees of polarization are much higher than in the other spectral ranges, and we benefit from the development of large instruments and sensitive receivers. This is why most of our knowledge on interstellar magnetic fields in our Galaxy (Reich, 1994; Heiles, 1996; Beck, 2001) and in external galaxies is based on polarized radio emission and its Faraday rotation (see also Beck, 2000; Beck, 2002). To detect extended features and achieve high resolution, data from interferometric (synthesis) and single dish telescopes have to be combined.

\section{Radio Waves Are Tracers of Magnetic Fields}

... simply the best ones. Interstellar magnetic fields are illuminated by cosmic-ray electrons emitting synchrotron radiation, the dominant contribution to the diffuse radio continuum emission at centimeter and decimeter wavelengths. Synchrotron emission is intrinsically highly linearly polarized, $70-75 \%$ in a completely regular magnetic field. The observable degree of polarization in galaxies is reduced by a contribution of unpolarized thermal emission which may dominate in starforming regions, by Faraday depolarization (Sokoloff et al., 1998) and by geometrical depolarization within the beam. A map of the total nonthermal intensity (Fig. 1) reveals the strength of the total interstellar magnetic fields in the plane of the sky, while the polarized intensity and

(C) 2018 Kluwer Academic Publishers. Printed in the Netherlands. 
polarization angle (Figs. 2 and 3) reveal the strength and structure of the resolved regular fields in the plane of the sky. The orientation of polarization vectors is changed in a magneto-ionic medium by Faraday rotation which is generally small below about $\lambda 6 \mathrm{~cm}$ so that the $\mathbf{B}-$ vectors (i.e. the observed $\mathbf{E}$-vectors rotated by $90^{\circ}$ ) directly trace the orientation of the regular fields in the sky plane.

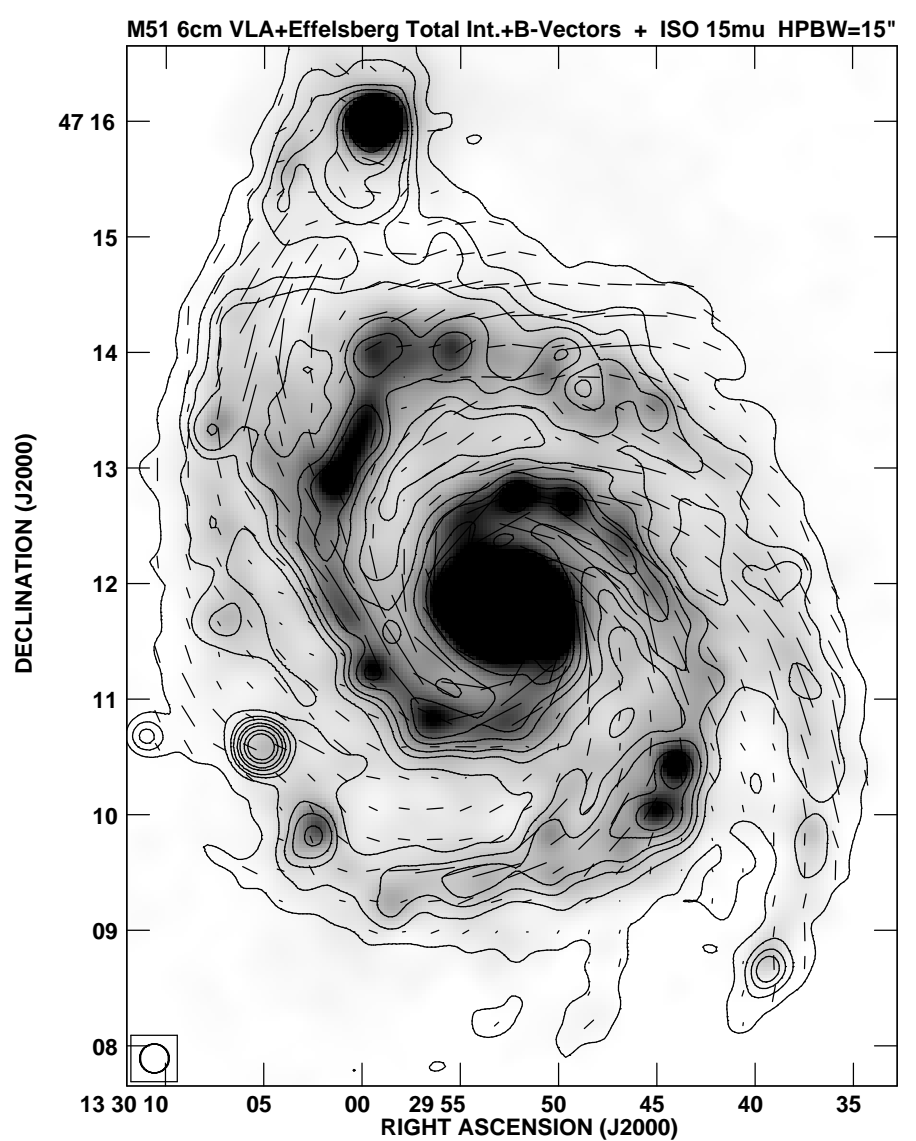

Figure 1. Total radio continuum intensity (contours) and B-vectors of polarized intensity of M51 at $15^{\prime \prime}$ resolution, combined from VLA and Effelsberg observations at $\lambda 6 \mathrm{~cm}$. The grey-scale ISOCAM image shows the $\lambda 15 \mu \mathrm{m}$ emission (kindly provided by M. Sauvage), smoothed to $15^{\prime \prime}$ resolution. (Beck, unpublished)

\section{Magnetic fields Are Strong}

... much stronger than most people think. The average strength of the total $\left\langle B_{\mathrm{t}, \perp}\right\rangle$ and the resolved regular field $\left\langle B_{\mathrm{reg}, \perp}\right\rangle$ in the plane of the sky can be derived from the total and polarized radio synchrotron 
intensity, respectively, if energy-density equipartition between cosmic rays and magnetic fields is assumed (Beck et al., 1996).

In our Galaxy the accuracy of the equipartition assumption can be tested, because we have independent information from in-situ measurements about the local cosmic-ray energy density and from $\gamma$-ray data about their distribution. Combination of the radio synchrotron emission, the local cosmic-ray electron density and diffuse continuum $\gamma$-rays yields a local strength of the total field of $6 \pm 1 \mu \mathrm{G}$ (Strong et al., 2000), the same value as derived from energy equipartition (Berkhuijsen in Beck, 2001). Even the scale length of the radial variation of the equipartition field strength is similar to that in Strong et al. (2000).

The mean equipartition strength of the total field for a sample of 74 spiral galaxies (derived from their total radio flux densities) is $\left\langle B_{\mathrm{t}}\right\rangle=$ $9 \mu \mathrm{G}$, ranging between $\left\langle B_{\mathrm{t}}\right\rangle \simeq 6 \mu \mathrm{G}$ in radio-faint galaxies like $\mathrm{M} 31$ and M 33, and $\simeq 15 \mu \mathrm{G}$ in grand-design galaxies like M 51, M 83 and NGC 6946. In prominent spiral arms the total field strength is 20$25 \mu \mathrm{G}$, up to $40 \mu \mathrm{G}$ in nuclear regions (Sect. 7.3), and even $1 \mathrm{mG}$ in molecular clouds compressed by supernova shocks (Brogan et al., 2000), in compact cloud cores (Myers and Goodman, 1988; Uchida et al., 2001) and in filaments near the Galactic center (Reich, 1994; Yusef-Zadeh et al., 1996).

\section{Magnetic Fields Are Dynamically Important}

... more important than most people think. A standard approach to estimate the dynamical importance of various competing forces is to compare the corresponding energy densities (Table 1). Note, however, that the dynamical effects of magnetic fields are anisotropic due to their vector nature. NGC 6946 (Fig. 2) is a useful standard spiral galaxy because it has no companion, no strong density waves, no active nucleus and has been observed in many spectral ranges. The average total equipartition field strength in the inner disk (within $4 \mathrm{kpc}$ radius) is $B_{t} \simeq 18 \mu \mathrm{G}$ (using the map of synchrotron emission by Walsh et al., $2002)$ and the average density of the warm ionized gas $\left(T \simeq 10^{4} \mathrm{~K}\right)$ is $n_{e} \simeq 0.5 \mathrm{~cm}^{-3}$ (using their map of thermal radio emission and a filling factor of $5 \%$ ). The map of the total neutral gas (molecular + atomic, where the molecular gas dominates until about $5 \mathrm{kpc}$ radius) yields a mean number density (within a disk of $4 \mathrm{kpc}$ radius and $100 \mathrm{pc}$ height) of $n \simeq 20 \mathrm{H}$ atoms $\mathrm{cm}^{-3}$. The turbulent velocity of the neutral gas is assumed to be $v_{\text {turb }}=7.5 \mathrm{~km} / \mathrm{s}$, as for the cold neutral gas in our Galaxy (Kalberla and Kerp, 1998), and the global rotation velocity

$v_{\text {rot }}=170 \mathrm{~km} / \mathrm{s}$. For the cosmic rays we know the slope of the energy 
Table I. Average energy densities $\epsilon$ (in $10^{-12} \mathrm{erg} \mathrm{cm}^{-3}$ ) in the ISM of the inner disk ( $\leq 4 \mathrm{kpc}$ radius) of NGC 6946

\begin{tabular}{lllr}
\hline Magnetic field (equip.) & $\epsilon_{B_{\mathrm{t}}^{2}}$ & $B^{2} / 8 \pi$ & 13 \\
Cosmic rays (equip.) & $\epsilon_{\mathrm{CR}}$ & $C \int E^{-2 \alpha} d E$ & 13 \\
Warm ionized medium & $\epsilon_{\mathrm{WIM}}$ & $\frac{3}{2} n_{e} k T$ & 1 \\
Turbulent cloud motion & $\epsilon_{\mathrm{turb}}$ & $\frac{1}{2} \rho v_{\mathrm{turb}}^{2}$ & 10 \\
Global gas rotation & $\epsilon_{\mathrm{rot}}$ & $\frac{1}{2} \rho v_{\mathrm{rot}}^{2}$ & 5000 \\
\hline
\end{tabular}

spectrum $\left(N[E] \propto N_{0} E^{-2 \alpha-1} d E\right)$ from the observed synchrotron spectral index $\alpha$, but no information on the absolute particle number $N_{0}$ is available so that we have to rely on the validity of energy equipartition with the magnetic fields.

The ratio $\beta$ between the thermal and magnetic energy densities of the warm ionized medium (WIM) is only $\simeq 0.1$. The hot ionized gas and the warm neutral gas are believed to be in pressure equilibrium with the WIM so that their $\beta$ should be similar. (The thermal energy of the cold gas is negligible.) A low thermal contribution to the total gas pressure was also found in the local Galactic ISM (Jenkins and Tripp, 2001; Jenkins, this volume). A significant fraction of the diffuse ISM must be unstable, giving rise to gas flows. From X-ray and radio continuum observations in the halo of the spiral galaxy M 83, Ehle et al. (1998) derived a similarly low value of $\beta$. The magnetic field dominates thermal processes in the disk and in the halo of galaxies.

\section{Magnetic Fields Drive Star Formation}

Turbulent motion of neutral gas and total magnetic fields in the inner disk of NGC 6946 are roughly in energy equipartition (Table 1) (but see Sect. 6). This fact supports the idea that magnetic fields are anchored in the (partly ionized) envelopes of gas clouds (Beck, 1991). This also explains the tight radio-infrared correlation (Niklas and Beck, 1997; Walsh et al., 2002). Between the clouds, field loops can be generated by the Parker instability which can drive the galactic dynamo (Hanasz and Lesch, 1998; Moss et al., 1999; Hanasz et al., this volume).

Interstellar magnetic fields affect the motion of small gas clouds (Elmegreen, 1981) and their collision rate. The importance of internal fields for the evolution of clouds is generally accepted, though not understood (Mestel and Paris, 1984; Myers and Goodman, 1988; Crutcher, 
1999; Heitsch et al., 2001). However, little is known about the influence of magnetic fields in the ISM on general properties of star formation.

Stability of cloud cores depends also on the fractional ionization by cosmic rays, and the number density of cosmic rays in the ISM is controlled by the interstellar magnetic fields. A larger fractional ionization allows a better coupling of the fields to the gas (weaker ambipolar diffusion) and thus stronger internal fields. As a result, the field strength inside and outside of the clouds should be correlated.

The star formation rate $S F R$ is known to relate non-linearly with gas density $\rho$, the Schmidt law $S F R \propto \rho^{\mathrm{n}}$, with $n \simeq 1.5$ (Wong and Blitz, 2002). Hence, the star formation efficiency $(S F E \propto S F R / \rho)$ increases with gas density. As the total field strength $B_{t}$ in galaxies is known to scale globally with $\rho$ as $B_{t} \propto \rho^{0.5}$ (Niklas and Beck, 1997), a relation $S F E \propto B_{t}^{\mathrm{x}}$ could well be the cause of the non-linearity. The observational data are still poor (Vallée, 1994). The supernova-driven ISM model including magnetic fields by Gazol-Patiño and Passot (1999) indeed predicts a significant increase of the average star-formation rate with field strength. Furthermore, strong fields may shift the stellar mass spectrum towards the more massive stars (Mestel, 1994).

\section{Do Magnetic Fields Dominate in Outer Galaxies ?}

Most observable quantities in galactic disks have exponential distributions. For NGC 6946 the scale length of the synchrotron emission is $l_{\text {syn }}=3.9 \pm 0.1 \mathrm{kpc}$ (radial range $3-8 \mathrm{kpc}$ ). The synchrotron disk is a combination of the distributions of cosmic-ray electrons and total magnetic fields. In case of equipartition between these two components, the scale length of the total magnetic field is $l_{\mathrm{B}}=(3+\alpha) l_{\mathrm{syn}} \simeq$ $4 l_{\text {syn }} \simeq 16 \mathrm{kpc}$, and the scale lengths of the magnetic energy density and of the cosmic rays are $l_{\mathrm{B}^{2}}=l_{\mathrm{CR}} \simeq \mathbf{8} \mathbf{k p c}$. All other energy densities in Table 1 fall off with the scale length of the neutral gas $l_{\rho}=3.2 \pm 0.1 \mathrm{kpc}$ if $T, v_{\text {turb }}$ and $v_{\text {rot }}$ are radially constant, or with a smaller scale length if one of these parameters decreases with radius.

The scale length of the distribution of supernova remnants (the sources of cosmic rays) in NGC 6946 is only $\simeq 2 \mathrm{kpc}$ (Sasaki et al., this volume) so that a large diffusion length is needed to reach equipartition also at large radii (see the cosmic-ray propagation model by Breitschwerdt et al., 2002). A large value of $l_{\mathrm{CR}}$ is also required to explain the distribution of Galactic $\gamma$-rays (Strong et al., 2000).

As a consequence of the different scale lengths $\beta=\epsilon_{\text {turb }} / \epsilon_{\mathrm{B}^{2}}$ decreases with radius: The dominance of the magnetic field increases towards large radii. It is however hard to understand why the magnetic 
field energy density is larger that of the turbulent motions. This can be in conflict with turbulent generation of interstellar magnetic fields which predicts energy equipartition between turbulent motions and magnetic fields, and hence $l_{\mathrm{B}^{2}}=l_{\rho}$ (if $v_{\text {turb }} \simeq$ const) and $l_{\text {syn }}<l_{\rho}$. Radial diffusion of the magnetic field (Priklonsky et al., 2000), field connections through the wind-driven halo (Breitschwerdt et al., 2002) or a supra-equipartition turbulent dynamo (Belyanin et al., 1993) are possible explanations.

In the outermost parts of galaxies the magnetic field energy density may even reach the level of global rotational gas motion. If so, the rotation curves at large radii could be affected by magnetic fields, as discussed by Battaner and Florido (2000). Field strengths in the outer parts of galaxies are difficult to measure. Synchrotron emission is weak, but Faraday rotation of polarized background sources can be measured out to large radii. Han et al. (1998) found evidence for regular fields in M31 at $25 \mathrm{kpc}$ radius of similar strength as in the inner disk. More detailed studies in a number of galaxies are required.

\section{Magnetic Fields Act Everywhere}

\subsection{Spiral Arms}

Maps of the total radio emission and ISOCAM maps of the mid-infrared dust emission (Fig. 1) reveal a surprisingly close connection (Frick et al., 2001; Walsh etal., 2002), suggesting a coupling of the total magnetic field to the warm dust mixed with cool gas $\left(B_{t} \propto \rho^{0.5}\right.$, Niklas and Beck, 1997). Strongest total fields generally coincide with highest emission from dust and gas in the spiral arms (Fig. 1), whereas the polarized emission is weak in the arms due to field tangling by turbulent motions or by supernova shock fronts. Radio polarization observations show that in most galaxies the regular field follows the spiral structure seen in the stars and the gas, though generally offset, sometimes forming magnetic spiral arms between the gaseous arms (Fig. 2). Here the energy density of the regular field alone $(\simeq 10 \mu \mathrm{G})$ is comparable to that of the neutral interarm gas $\left(\rho \simeq 5 \mathrm{~cm}^{-3}\right)$.

In galaxies with strong density waves (like M 51) the regular field is strong at the positions of the dust lanes on the inner edge of the spiral arms. However, the arm-interarm contrast of the regular field is low. The strong field may modify the gas flow and the compression ratio.

The observation of large-scale patterns in Faraday rotation measures proves that the regular field in galaxies has a coherent direction and is not generated by compression or stretching of irregular fields in gas 
flows. The dynamo mechanism is able to generate and preserve coherent magnetic fields of spiral shape (Beck et al., 1996; Moss et al., 1999). The observed alignment of magnetic pitch angles with those of the gaseous arms can be achieved by inclusion of the shear flow around spiral arms (von Linden et al., 1998). Future dynamo models should address the interaction between dynamo wave and density wave.

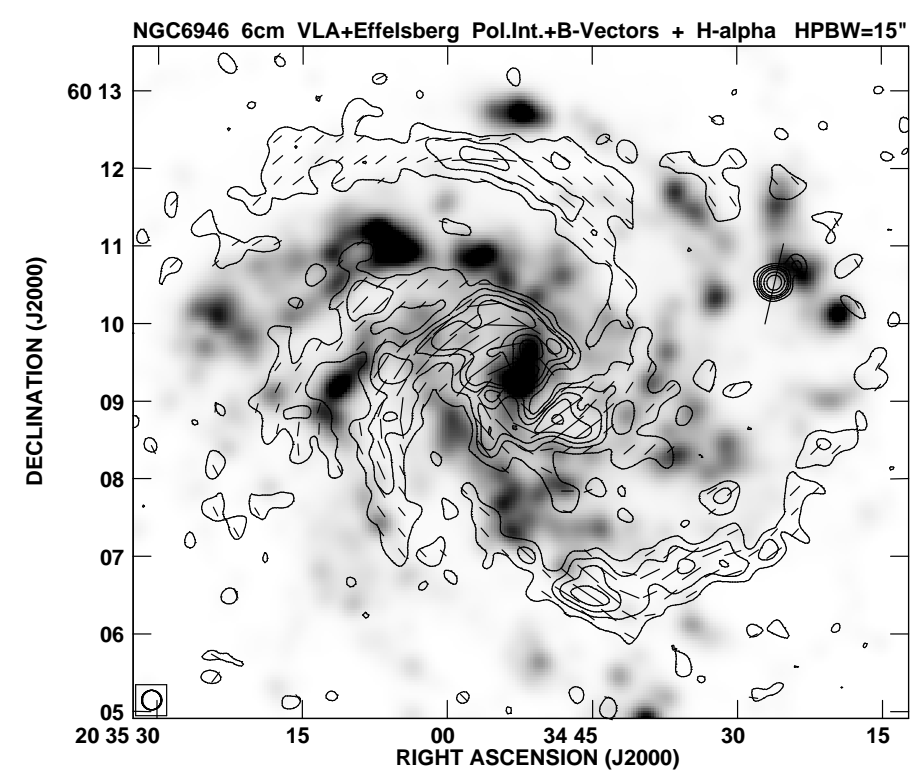

Figure 2. Polarized radio intensity (contours) and B-vectors of polarized intensity of NGC 6946 at $15^{\prime \prime}$ resolution, combined from VLA and Effelsberg observations at $\lambda 6 \mathrm{~cm}$. The grey-scale image shows the $\mathrm{H} \alpha$ emission (kindly provided by A. Ferguson), smoothed to $15^{\prime \prime}$ resolution. (From Beck and Hoernes, 1996)

\subsection{BARS}

Average radio intensity, radio luminosity and star-formation activity in barred galaxies correlate with relative bar length (Beck et al., 2002). In the best-studied case, NGC 1097, the general similarity of the $B-$ vectors (Fig. 3) and gas streamlines around the bar as obtained in simulations is striking (Beck et al., 1999). The field lines are a tracer of the sheared flow pattern in the sky plane which is otherwise unobservable. Remarkably, the optical image of NGC 1097 shows dust filaments outside the bar which are aligned with the field.

The bar's major dust lane is believed to indicate the shock front. However, the region of strongest shear in NGC 1097 (where the field changes its direction abruptly) is located $\simeq 1 \mathrm{kpc}$ in front of the dust lane (Fig. 3), in conflict with the hydrodynamical models. Furthermore, 
the regular field is only weakly compressed in the bar. Either the field decouples from the gas (at least from its diffuse component) and thus avoids the gas shock, or there is no shock at all. Detailed observations of the velocity field are required. Moss et al. (2001) used velocity shear and dynamo action to model the field configuration, but a MHD model including the back-reaction of the field onto the gas flow is still needed.

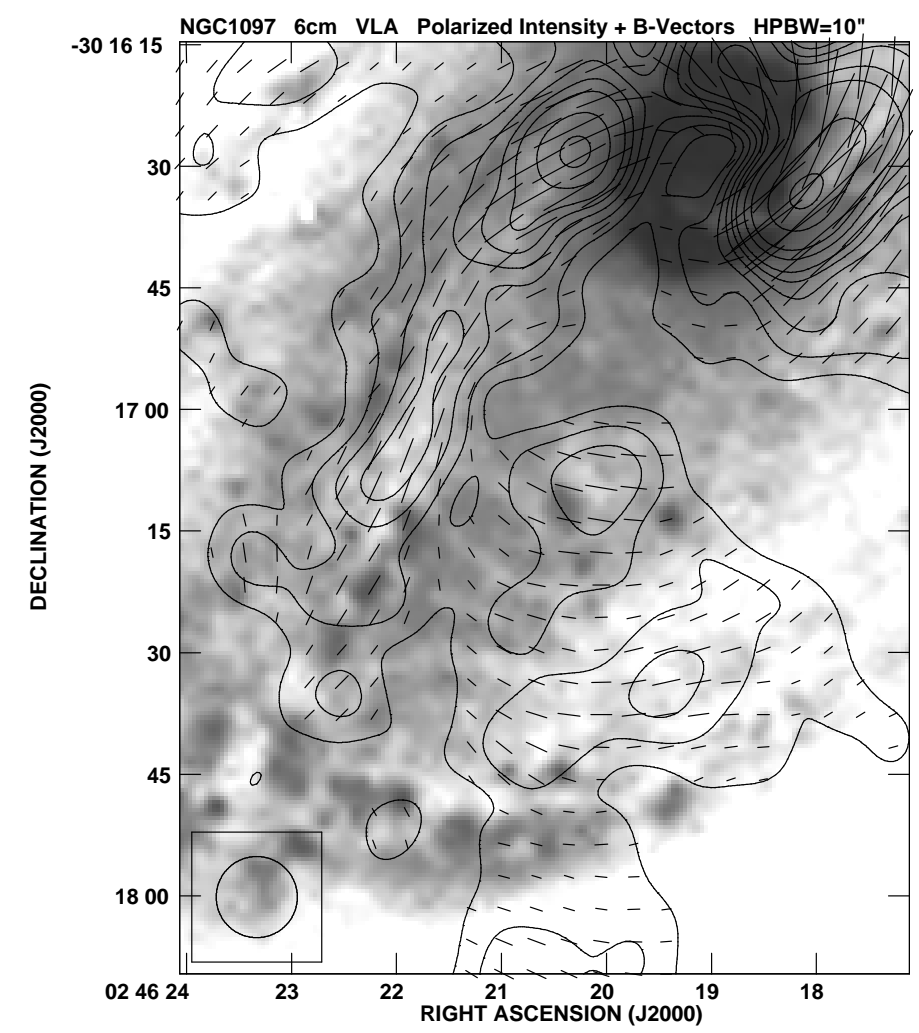

Figure 3. Polarized radio intensity of NGC 1097 (contours) and B-vectors of polarized intensity at $10^{\prime \prime}$ resolution, observed with the VLA at $\lambda 6 \mathrm{~cm}$. The grey-scale image shows an optical image from the Cerro Tololo Observatory (kindly provided by H. Arp). (From Moss et al., 2001)

\subsection{NuCleAR REgions}

Many barred galaxies have circumnuclear rings, sites of ongoing intense star formation, sometimes with an active nucleus in the center. Radio polarization maps of these inner regions (Beck et al., 2002) revealed strong regular fields (up to $40 \mu \mathrm{G}$ ) with spiral patterns and large pitch angles, accompanied by spiral dust filaments visible on optical images. Magnetic stress in the circumnuclear ring can drive mass inflow to feed the active nucleus, as suggested for NGC 1097 (Beck et al., 1999). 


\subsection{HaLOS}

In edge-on galaxies the observed field orientations are mainly parallel to the disk (Dumke et al., 1995). A prominent exception is NGC 4631 with the brightest and largest halo observed so far (Beck, 2000), composed of vertical magnetic spurs connected to star-forming regions in the disk (Golla and Hummel, 1994). The field is probably dragged out by a strong galactic wind. The magnetic energy density in the halo of, e.g. M 83, exceeds that of the hot gas (Ehle et al., 1998). Halo magnetic fields are important for the propagation of cosmic rays, the formation of a galactic wind (Breitschwerdt et al., 2002) and the stability of gas filaments (Tüllmann et al., 2000; Dettmar, this volume).

\section{Outlook}

Much has been learnt about the global properties of interstellar magnetic fields, but their detailed structure on scales below about $100 \mathrm{pc}$ is still unclear. Polarization observations in our Galaxy trace structures on pc and sub-pc scales (e.g. Uyanıker et al., 1999; Gaensler et al., 2001). Future radio polarization observations with increased sensitivity and resolution (Extended VLA, Square Kilometer Array) will show the full wealth of magnetic structures in galaxies.

\section{References}

Battaner, E., and Florido, E.: 2000, Fund. Cosmic Phys. 21, 1-154.

Beck, R.: 1991, Astron. Astrophys. 251, 15-26.

Beck, R.: 2000, Phil. Trans. R. Soc. Lond. A 358, 777-796.

Beck, R.: 2001, Space Science Reviews 99, 243-260.

Beck, R.: 2002, in E. Athanassoula, A. Bosma and R. Mujica (eds.), Disks of Galaxies, ASP Conf. Ser. 275, pp. 331-342.

Beck, R., and Hoernes, P.: 1996, Nature 379, 47-49.

Beck, R., Brandenburg, A., Moss, D., Shukurov, A., and Sokoloff, D.: 1996, Ann. Rev. Astron. Astrophys. 34, 155-206.

Beck, R., Ehle, M., Shoutenkov, V., Shukurov, A., and Sokoloff, D.: 1999, Nature 397, 324-327.

Beck, R., Shoutenkov, V., Ehle, M., et al.: 2002, Astron. Astrophys. 391, 83-102.

Belyanin, M., Sokoloff, D., and Shukurov, A.: 1993, Geophys. Astrophys. Fluid Dyn. 68, 227-261.

Breitschwerdt, D., Dogiel, V.A., and Völk, H.J.: 2002, Astron. Astrophys. 385, 216238.

Brogan, C.L., Frail, D.A., Goss, W.M., and Troland, T.H.: 2000, Astrophys. J. 537, 875-890.

Crutcher, R.M.: 1999, Astrophys. J. 520, 706-713. 
Dumke, M., Krause, M., Wielebinski, R., and Klein, U.: 1995, Astron. Astrophys. 302, 691-703.

Ehle, M., Pietsch, W., Beck, R., and Klein, U.: 1998, Astron. Astrophys. 329, 39-54.

Elmegreen, B.G.: 1981, Astrophys. J. 243, 512-525.

Frick, P., Beck, R., Berkhuijsen, E.M., and Patrickeyev, I.: 2001, Mon. Not. R. Astron. Soc. 327, 1145-1157.

Gaensler, B.M., Dickey, J.M., McClure-Griffiths, N.M., et al.: 2001, Astrophys. J. 549, 959-978.

Gazol-Patiño, A., and Passot, T.: 1999, Astrophys. J. 518, 748-759.

Golla, G., and Hummel, E.: 1994, Astron. Astrophys. 284, 777-792.

Han, J.L., Beck, R., and Berkhuijsen, E.M.: 1998, Astron. Astrophys. 335, 11171123.

Hanasz, M., and Lesch, H.: 1998, Astron. Astrophys. 332, 77-87.

Heiles, C.: 1996, in W. G. Roberge and D. C. B. Whittet (eds.), Polarimetry of the Interstellar Medium, ASP Conf. Ser. 97, pp. 457-474.

Heitsch, F., Mac Low, M., and Klessen, R.S.: 2001, Astrophys. J. 547, 280-291.

Jenkins, E.B., and Tripp, T.M.: 2001, Astrophys. J. Suppl. 137, 297-340.

Kalberla, P.M.W., and Kerp, J.: 1998, Astron. Astrophys. 339, 745-758.

von Linden, S., Otmianowska-Mazur, K., Lesch, H., and Skupniewicz, G.: 1998, Astron. Astrophys. 333, 79-91.

Mestel, L.: 1994, in D. Lynden-Bell et al. (eds.), Cosmical Magnetism, Kluwer, Dordrecht, pp. 181-211.

Mestel, L., and Paris, R.B.: 1984, Astron. Astrophys. 136, 98-120.

Moss, D., Shukurov, A., and Sokoloff, D.: 1999, Astron. Astrophys. 343, 120-131.

Moss, D., Shukurov, A., Sokoloff, D., Beck, R., and Fletcher, A.: 2001, Astron. Astrophys. 380, 55-71.

Myers, P.C., and Goodman, A.A.: 1988, Astrophys. J. 326, L27-L30.

Niklas, S.: 1995, Ph.D. Thesis, University of Bonn.

Niklas, S., and Beck, R.: 1997, Astron. Astrophys. 320, 54-64.

Priklonsky, V.I., Shukurov, A., Sokoloff, D., and Soward, A.: 2000, Geophys. Astrophys. Fluid Dyn. 93, 97-114.

Reich, W.: 1994, in R. Genzel and A.I. Harris (eds.), The Nuclei of Normal Galaxies, Kluwer, Dordrecht, pp. 55-62.

Sokoloff, D. D., Bykov, A. A., Shukurov, A., Berkhuijsen, E. M., Beck, R., and Poezd, A.D.: 1998, Mon. Not. R. Astron. Soc. 299, 189-206 and Mon. Not. R. Astron. Soc. 303, 207-208 (Erratum).

Strong, A. W., Moskalenko, I. V., and Reimer, O.: 2000, Astrophys. J. 537, 763-784.

Tüllmann, R., Dettmar, R.J., Soida, M., Urbanik, M., and Rossa, J.: 2000, Astron. Astrophys. 364, L36-L41.

Uchida, K.I., Fiebig, D., and Güsten, R.: 2001, Astron. Astrophys. 371, 274-286.

Uyanıker, B., Fürst, E., Reich, W., Reich, P., and Wielebinski, R.: 1999, Astron. Astrophys. Suppl. 138, 31-45.

Vallée, J.P.: 1994, Astrophys. J. 433, 778-779.

Walsh, W., Beck, R., Thuma, G., Weiss, A., Wielebinski, R., and Dumke, M.: 2002, Astron. Astrophys. 388, 7-28.

Woltjer, L.: 1967, in: H. van Woerden (ed.), Radio Astronomy and the Galactic System, Reidel, Dordrecht, pp. 479-485.

Wong, T., and Blitz L.: 2002, Astrophys. J. 569, 157-183.

Yusef-Zadeh, F., Roberts, D. A., Goss, W. M., Frail, D. A., and Green, A. J.: 1996, Astrophys. J. 466, L25-L29. 